\title{
A Cellular Automaton Model of Brain Tumor Treatment and Resistance
}

\author{
JONATHAN E. SCHMITZ ${ }^{\mathrm{a}}$, ANURAAG R. KANSAL ${ }^{\mathrm{b}}$ and SALVATORE TORQUATO ${ }^{\mathrm{a}, \mathrm{c}, *}$ \\ ${ }^{\mathrm{a}}$ Department of Chemistry, Princeton University, Princeton, NJ 08544, USA $;{ }^{\mathrm{b}}$ Department of Chemical Engineering, Princeton University, Princeton, \\ NJ 08544, USA; ${ }^{\mathrm{c}}$ Princeton Material Institute, Princeton University, Princeton, NJ 08544, USA \\ (Received 22 May 2002; In final form 2 January 2003)
}

\begin{abstract}
We have extended an automaton model of brain tumor growth to study the effects of treatment. By varying three treatment parameters, we can simulate tumors that display clinically plausible survival times. Much of our work is dedicated to heterogeneous tumors with both treatment-sensitive and treatment-resistant cells. First, we investigate two-strain systems in which resistant cells are initialized within predominantly sensitive tumors. We find that when resistant cells are not confined to a particular location, they compete more effectively with the sensitive population. Moreover, in this case, the fraction of resistant cells within the tumor is a less important indicator of patient prognosis when compared to the case in which the resistant cells are scattered throughout the tumor. In additional simulations, we investigate tumors that are initially monoclonal and treatment-sensitive, but that undergo resistance-mutations in response to treatment. Here, the tumors with both very frequent and very infrequent mutations develop with more spherical geometries. Tumors with intermediate mutational responses exhibit multi-lobed geometries, as mutant strains develop at localized points on the tumors' surfaces.
\end{abstract}

Keywords: Glioblastoma multiforme; Chemotherapy; Drug resistance; Heterogeneous tumors; Computational modeling; Biosystem

\section{INTRODUCTION}

The most severe grade of astrocytic brain tumor, glioblastoma multiforme (GBM) is one of the deadliest forms of human cancer. The typical approach for treating GBM involves surgical resection of as much of the tumor as possible, followed by radiation treatment and chemotherapy (Holland, 2000). Despite these techniques, long-term prognoses are still rather bleak. Drug resistant tumor cells are a formidable obstacle in this regard. Within a single tumor of monoclonal origin, there can develop multiple sub-populations, each of which may be characterized by different growth-rates and treatment susceptibilities (Yung et al., 1982; Paulus and Peiffer, 1989; Berkman et al., 1992; Coons and Johnson, 1993).

Treatment resistance is itself a complex phenomenon. There is no single cause of resistance, and many biochemical aspects of it are poorly understood. Chemoresistant strains can either be resistant to a single drug or drug family (individual resistance), or they can be resistant to an array of agents (multidrug/pleotropic resistance) (Bredel, 2001). Cellular mechanisms behind multidrug resistance include increased chemical efflux and/or decreased chemical influx, such as with Pglycoprotein (P-gp)-mediated drug resistance (Endicott and Ling, 1989; German, 1996).

Complicating the situation further, resistance can arise at variable times during tumor development. Some tumors are resistant to chemotherapy from the onset. This has been described as inherent resistance, because it exists before chemotherapeutic drugs are ever introduced. In other cases, however, treatment initially proves successful, and only later does the tumor, prove resistant. This is an example of acquired resistance, as it develops in response to treatment (Bredel, 2001). There are at least two possible mechanisms for this type of tumor behavior. Acquired resistance may result from a small number of resistant cells that are gradually selected for throughout the course of treatment. At the same time, there is also evidence suggesting that chemotherapeutic agents may induce genetic or epigenetic changes within tumor cells, leading to a resistant phenotype. For example, Poppenborg et al. (1997) have reported that a glioma cell line pretreated with cis-diamminedichloroplatinum(II) (cisplatin) shows

*Corresponding author. E-mail: torquato@princeton.edu 
increased cisplatin resistance compared to untreated cells. Their data suggest that the resistance is mediated by induced mutations and up-regulated glutathione levels. Other studies indicate that chemotherapy may increase cellular levels of P-gp mRNA and protein in various forms of human cancer (Chadhary and Roninson, 1993; Gekeler et al., 1994). A tumor's response to radiation therapy can also depend on underlying genetic factors. A cell's inherent radio-resistance may stem from the efficiency of DNA repair mechanisms in sublethally damaged cells (Gerweck et al., 1977; Kayama et al., 1991; Zhang et al., 1993).

In response to this dilemma, theoreticians have developed a number of mathematical models to explain tumor treatment and resistance. Brain tumors are, in many ways, ideal candidates for theoretical modeling. Their rapid growth and resilience suggests that many may be investigated as self-organizing, dynamic systems (Kraus and Wolf, 1993; Deisboeck et al., 2001). Mathematical approaches to tumor treatment offer a perspective that current in vivo/in vitro techniques cannot. Some of the earliest work toward understanding drug resistance comes from Coldman and Goldie (1979). They proposed a model which links a tumor's drug sensitivity to its rate of spontaneous resistance mutations. They hypothesized that tumors may experience mutations that lead to resistance against drugs to which they have not been exposed previously. The following differential equation was utilized to relate the mean volume of a resistant strain to total tumor volume:

$$
\frac{\mathrm{d} \mu}{\mathrm{d} t}=\frac{\mu}{N}+\alpha\left(1-\frac{\mu}{N}\right)
$$

In this equation, $\mu$ is the mean volume of the resistant strain, $N$ is the total volume of the tumor and $\alpha$ is the mutation rate per cell generation. With the assumption that back mutations (resistant to sensitive) may occur, the expected number of resistant cells for a tumor of size $N$ is given by

$$
\mu=\frac{1}{2}\left(1-N^{-2 \alpha}\right) N
$$

Later work built upon this topic to consider such issues as the effectiveness of various drug protocols, as well as the outcome of treatment on tumors with both actively dividing and quiescent cells (Coldman and Goldie, 1985; 1986).

Additional investigations have likewise studied the effects of treatment on heterogeneous tumors. Tracqui et al. (1995) considered chemotherapy in light of the invasive ability of glioma cells. With a simple deterministic approach, they found that the effects of treatment in one patient do not agree with standard tumor growth models, unless a resistant sub-population is also considered. In 1998, Panetta relied upon sets of coupled ordinary differential equations to model the size of two sub-populations in time:

$$
\begin{gathered}
\frac{\mathrm{d} x}{\mathrm{~d} t}=\left[r_{1}-d_{1}(t)\right] x \\
\frac{\mathrm{d} y}{\mathrm{~d} t}=b_{1} d_{1}(t) x+\left[r_{2}-d_{2}(t)\right] y .
\end{gathered}
$$

In these equations, $x$ represents the sensitive cell population, $y$ represents the resistant cell population, $r_{1}$ and $r_{2}$ are their respective growth-rates and $d_{1}$ and $d_{2}$ are their respective drug sensitivities. Panetta's investigation touched upon several topics, including the development of treatment protocol and the delivery of combination chemotherapy. Birkhead et al. (1987) similarly employed coupled ordinary differential equation in their study, which considered both cycling and resting tumor cells. Both classifications included treatment-sensitive and treatment-resistant cells. Sensitive cells could convert to resistant cells, while cycling and resting cells could interconvert. In several scenarios, the treatment routine and the cellular sensitivity were varied to illustrate general principles of successful chemotherapy. In other work, Costa et al. (1995) and Boldrini (2000) attempt to formulate effective treatment methodologies by analyzing tumor-systems from an optimization perspective.

In the more general context of clonal heterogeneity, Michelson et al. have proposed several models describing the emergence of sub-populations. They first developed a deterministic model to study the dynamics of inter-strain competition in both classically competitive and emergent environments (1987). This approach is an extension of a model initially proposed by Jansson and Revesz (1974). Later, Michelson et al. (1989) devised a stochastic analog to the first investigation. Here, processes were governed by a Fokker-Planck probability density function, for which solutions were generated numerically. They demonstrated that both the deterministic and stochastic models suggest a like picture of tumor dynamics. These studies did not specifically examine differential drug resistance among strains, but they are quite applicable to the topic. In a later paper, Michelson (1989) focused on treatment with an investigation of the P-gp pump, multidrug resistance, and its reversal.

These previous models have attempted to approach tumor treatment with a great deal of mathematical rigor. At the same time, one could envision certain ways by which their scope could be extended. With deterministic approaches, for example, the sets of equations that govern tumor behavior often do not correspond to the characteristics of individual tumor cells. An important goal of studying tumor development is to illustrate how their macroscopic traits stem from their microscopic properties. Another potential challenge is to formulate models that can be appreciated by as diverse an audience as possible. Ideally, the mathematical complexity that allows theoreticians to analyze subtle aspects of treatment should not be an obstacle for clinicians who treat GBM. 
A model that accounts for complex tumor behavior with relative mathematical ease could be valuable.

To this end, we have developed a three-dimensional automaton cell simulation of brain tumor treatment. In earlier work, we reported a four-parameter model that describes volumetric tumor proliferation (Kansal et al., 2000b). We will henceforth refer to this investigation as paper I. A second study (paper II) utilizes the simulation procedure of paper I to analyze heterogeneous tumors, in which sub-populations possess different growth-rates (Kansal et al., 2000a). In our present work, we take the four-parameter proliferation routine of papers I and II, and extend it with three additional parameters to simulate the effects of treatment, resistance and induced mutations. It has been some time since an automaton model of tumor treatment has been proposed. Düchting and Vogelsaenger (1985) touched upon chemotherapy as part of their automaton study of tumor growth. However, their cubic model only investigated small-scale tumors, and their focus on chemotherapy within the paper was brief. To the best of our knowledge, this is the first automaton investigation that focuses solely upon brain tumor treatment. Although the data here should not be extrapolated quantitatively to the behavior of real-life GBM, this work does suggest several qualitative trends in the dynamics of these tumors under the effects of treatments.

This paper is organized in the following manner. In the Methods section, we outline the simulation and its important features. The Results section then presents the computational data. Three separate case studies are considered here, each of which investigates the effects of treatment on a different post-surgical tumor-system: monoclonal tumors, two-strain tumors with resistant sub-populations and multi-strain tumors with induced mutations. The broader significance of the data is described in the Discussion and Conclusions. This section also describes intriguing avenues for future research in tumor modeling. We discuss both potential extensions of our current algorithm, as well as more general ways in which automaton simulations could advance in the future. Finally, a brief Appendix describes several computational issues of the current investigation.

\section{METHODS}

The algorithm employed in this investigation includes both a proliferation routine, as well as a newly-developed treatment routine. The proliferation routine was initially proposed in paper I; the current treatment model is an extension of it. In the following section, the entire simulation is described. We should emphasize, however, that the discussion of the proliferation routine is intended only to be a brief summary. For additional and more specific details, the reader is referred to paper I.

To begin, polyhedral automaton cells are generated through the three-dimensional Delaunay triangulation of space (Okabe et al., 1992; Torquato, 2002). Each isotropic automaton cell represents a cluster of several thousand biological cells. The simulation classifies the automaton cells into one of four types. Non-tumorous cells are designated as healthy and are inert. The outermost layer of the tumor consists of actively-dividing malignant cells, referred to as proliferative. Within the proliferative cells resides a layer of quiescent, non-proliferative cells. These cells can be viewed as existing in the $G_{0} / G_{1}$ arrested state of the cell cycle. Finally, at the center of the tumor, there exists of core of necrotic cells. Transitions from proliferative and non-proliferative to necrotic are governed by the nutritional requirements of the cell types.

Figure 1 depicts a cross sectional view of an idealized monoclonal tumor, with the proliferative, non-proliferative and necrotic zones highlighted. The tumor is nearly spherical in shape, and each of the three regions is concentric. The distances $\delta_{\mathrm{n}}$ and $\delta_{\mathrm{p}}$ are explained in the proceeding discussion of the algorithm. The overall tumor radius, $R_{\mathrm{t}}$, is depicted in Fig. 1; the reader is referred to the Appendix, part A for a description of how $R_{\mathrm{t}}$ is calculated, as there has been a slight change since papers I and II.

Our simulation depends upon seven parameters. Originally described in paper I, the four growth parameters ( $p_{0}, a, b$ and $R_{\max }$ ) reflect, respectively, the rate at which the proliferative cells divide, the nutritional needs of the non-proliferative and proliferative cells, and the response of the tumor to mechanical pressure within the skull. In addition to these terms, we now introduce three additional parameters for the treatment routine: $\gamma, \epsilon$ and $\phi$. The value of $\gamma$ reflects the proliferative cells' sensitivity at each instance of treatment. A high value of $\gamma$ corresponds to a sensitive tumor strain, while a low value corresponds to a resistant strain. Together, the values of $\gamma$ and $\epsilon$ determine the non-proliferative cells' treatment sensitivity. The parameter $\epsilon$ allows a different fraction of non-proliferative cells to die with every round of treatment compared to the proliferative cells. It reflects the differing susceptibility of cells in the arrested state (versus those in the proliferative state). Finally, $\phi$ governs the mutational response of the tumor to treatment-specifically it determines what fraction of cells undergo treatmentsensitivity mutations (i.e. changes in $\gamma$ ) with each round of treatment. All of the parameters and mathematical terms involved in the simulations are summarized in Table I.

At the onset of each simulation, a spherical tumor is initialized at a radius of $4 \mathrm{~mm} .^{\dagger}$ In GBM treatment,

\footnotetext{
${ }^{\dagger}$ Actually, the tumor is initialized as a mass of proliferative cells at a radius slightly less than $4 \mathrm{~mm}$. The algorithm is prerun for several days, so that the proper fraction of proliferative, non-proliferative, and necrotic cells can form. Day \#1 of the post-surgery simulation is denoted as the time at which the tumor's radius is $4 \mathrm{~mm}$.
} 


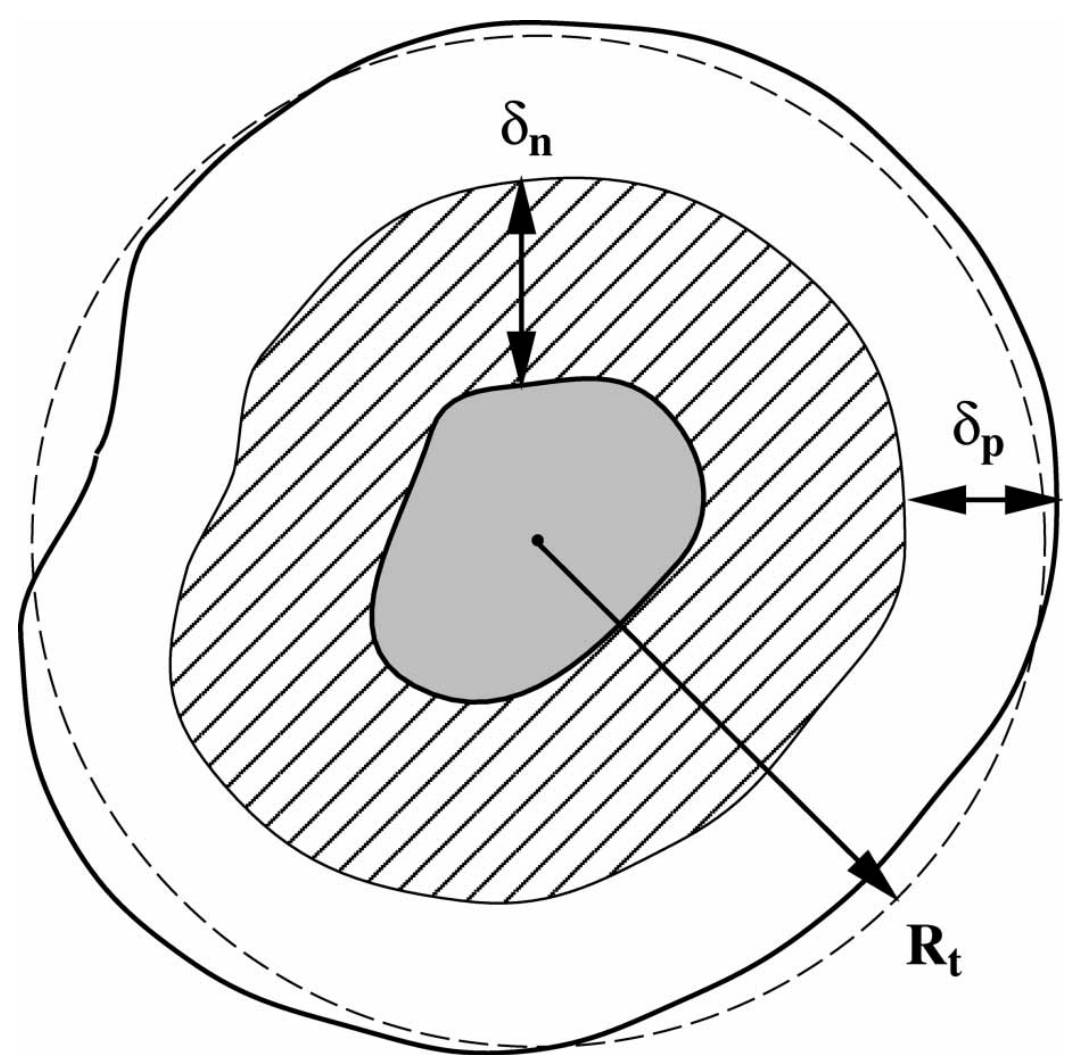

FIGURE 1 An idealized tumor. The inner gray region is composed of necrotic tissue. The cross-hatched layer is composed of living, quiescent cells (non-proliferative). It has a thickness $\delta_{n}$. The outer shell, with thickness $\delta_{p}$, is composed of proliferative cells.

surgery typically precedes chemotherapy and radiation. This size tumor is intended to represent a GBM tumor after successful surgical resection. Following paper I, we define the typical radius of a glial tumor at diagnosis as $18.5 \mathrm{~mm}$. An initial radius of $4 \mathrm{~mm}$ corresponds to approximately $1 \%$ of this diagnostic volume $(99 \%$ resection).

We must note that, by initializing these tumors as spheres, we are making a simplifying assumption as to the actual geometry of post-surgical tumors. In real-life conditions, resection would not leave behind a perfect sphere of cancerous tissue. Surgery could instead leave behind an alternate geometry, or a thin margin of cancerous cells surrounding the original tumor-site. Nevertheless, we feel for several reasons that our method of spherical initialization is suitable for the purposes of the investigation. For instance, one must consider the effects of a post-surgical collapse of the tissue that surrounded the tumor. Surgery does not leave behind a permanent hollow cavity in the brain; a thin margin of tumor cells would not remain a margin. Overall, post-surgical tumor modeling is restricted in that there is no ubiquitous geometry for brain tumors following resection. Due to this limitation, we focus here on studying more general principles of tumor growth and treatment, principles that are relevant regardless of initial cellular configurations (see "Discussion and Conclusions" section for specific examples).

Following tumor initialization, the proliferation/ treatment algorithm is discretized into time units that represent one day of real time. At each day, the simulation executes the following steps:

- Non-proliferative cells that are too far removed from the tumor's surface undergo cell death, converting to necrotic cells. The distance at which this transition occurs $\left(\delta_{\mathrm{n}}\right)$ is defined as:

$$
\delta_{\mathrm{n}}=a R_{\mathrm{t}}^{2 / 3},
$$

where $R_{\mathrm{t}}$ is the tumor radius and $a$ is the nonproliferative nutritional parameter. The twothirds power law reflects a surface-to-volume ratio due to nutrients diffusing through the tumor surface.

- Next, each proliferative cell will attempt to divide with a probability $p_{d}$, defined as:

$$
p_{d}=p_{0}\left(1-\frac{R_{\mathrm{t}}}{R_{\max }}\right),
$$

where $p_{0}$ is the base probability of division and $R_{\max }$ is the pressure response parameter. 
TABLE I Parameters and terms

Functions within the model (time dependent)

Average overall tumor radius (see Appendix)

Proliferative rim thickness (determines growth fraction)

Non-proliferative thickness (determines necrotic fraction)

Probability of division (varies with time and position)

Growth parameters

$P_{0} \quad$ Base probability of division, linked to cell-doubling time (0.192)

a Base necrotic thickness, controlled by nutritional needs $\left(0.42 \mathrm{~mm}^{1 / 3}\right)$

$b \quad$ Base proliferative thickness, controlled by nutritional needs $\left(0.11 \mathrm{~mm}^{1 / 3}\right)$

$R_{\max } \quad$ Maximum tumor extent, controlled by pressure response $(38 \mathrm{~mm})$

\section{Treatment parameters}

$\gamma \quad$ Governs the proliferative cells' response at each instance of treatment $(0.55-0.95)$

$\epsilon \quad$ Allows for different treatment responses between proliferative and non-proliferative cells $(0-0.4)$

$\phi \quad$ Fraction of surviving proliferative cells that mutate in response to treatment $\left(10^{-5}-10^{-2}\right)$

Other terms

$D_{\text {pro }} \quad$ Fraction of proliferative cells that die upon treatment; equivalent to $\gamma$

$D_{\text {non }} \quad$ Fraction of non-proliferative cells that die upon treatment; equivalent to $\gamma \epsilon$

$\beta \quad$ Volumetric fraction of living cells (proliferative and non-proliferative) belonging to secondary strain

Summarized here are definitions for the growth parameters, the treatmen parameters, and all other mathematical terms of the investigation. For each parameter, the number(s) listed in parentheses indicates the value or range of values that the parameter is assigned during the simulations. For all growth parameters, the values employed are based upon results from paper I.

- If a proliferative cell attempts to divide, it will search a radius $\delta_{\mathrm{p}}$ (the proliferative thickness) for a healthy cell, in which

$$
\delta_{\mathrm{p}}=b R_{\mathrm{t}}^{2 / 3}
$$

Here, $b$ is the proliferative nutritional parameter. Those cells that find space divide, taking care to ensure that division is continuous (i.e. into a neighbor cell). Cells that cannot divide become non-proliferative.

In the current investigation, the values of the four growth parameters are held constant. Following papers I and II, $p_{0}=0.192, a=0.42 \mathrm{~mm}^{1 / 3}, b=0.11 \mathrm{~mm}^{1 / 3}$ and $R_{\max }=38 \mathrm{~mm}$. The simulation proceeds through the above proliferative steps until every four week time-point, at which time the treatment routine is introduced:

- After the last round of cellular division, each proliferative cell is checked to see if it is killed by the treatment. The probability that a given proliferative cell dies, $D_{\text {pro }}$, is given by the relation

$$
D_{\text {pro }}=\gamma,
$$

where $\gamma$ is the proliferative treatment parameter. Its value can range from 0 to 1 . Since the proliferative rim adjoins non-tumorous tissue, dead proliferative cells are removed from the tumor: if a proliferative automaton cell is selected to die, it is converted to a healthy cell.

- Next, each non-proliferative cell is checked to see if it is killed. The probability of death for a given nonproliferative cell, $D_{\text {non, }}$, is given by

$$
D_{\text {non }}=\gamma \epsilon
$$

in which $\epsilon$ is the non-proliferative treatment parameter. The value of $\epsilon$ can also range between 0 and 1 , such that $D_{\text {non }}$ is a fraction of $D_{\text {pro }}$. As during the proliferation algorithm, a non-proliferative cell is converted to a necrotic cell upon death.

- Each surviving non-proliferative cell is now checked to see if it is has moved back within the proliferative thickness of a healthy cell (i.e. the tumor surface). If a healthy cell exists within this distance, the nonproliferative cell is converted back to a proliferative cell.

- Finally, all proliferative cells (including newlydesignated ones) are checked for mutations. For each automaton cell, there exists a probability, $\phi$, that its treatment resistance will change. When a cell is selected for mutation, a random number generator assigns it a new $\gamma$ value from 0 to 1 . The value of $\epsilon$ remains constant.

In real life, GBM treatment consists of both radiation therapy and chemotherapy. In this model, though, we do not distinguish between the separate effects of these two methods. The tumors' response to all treatment is captured by the treatment algorithm. Moreover, this response is assumed to be instantaneous at each four-week time point. Several previous models have likewise viewed treatment in this manner (Düchting and Vogelsaenger, 1985; Birkhead et al., 1987; Panetta, 1998). Panetta refers to this procedure as pulsed treatment. Although it simplifies the kinetics of drug diffusion, pulsed treatment corresponds to the manner in which clinical information is collected. Just as medical data is collected in a discrete fashion, it is natural for treatment to be modeled by a discrete method.

As indicated above, $\gamma$ and $\epsilon$ can assume a continuum of values from 0 to 1 . The simulation does not view resistance in an on/off sense. In a more realistic manner, cells can undergo varying degrees of death at each instance of treatment. We should discuss briefly the constraint $D_{\text {non }} \leq D_{\text {pro }}$. Since they exist in a growtharrested state of the cell cycle, non-proliferative cells should be less susceptible to treatment than their proliferative counterparts. In a condition known as phase resistance, quiescent cells can avoid the lethal effects of chemotherapy (Shah and Schwartz, 2001). Birkhead et al., (1987) viewed phase resistance in an extreme sense, as the model only considered the consequences of treatment 
on actively-dividing cells. We instead choose to include the term $\epsilon$ so that our simulation can incorporate nonproliferative cell death, albeit reduced. The tumor behavior in Birkhead et al.'s model corresponds to the special case in our simulation where $\epsilon=0$.

The reader should note that the parameter $\phi$ does not represent the probability of single real cell mutating upon treatment. The automaton cells in this simulation represent clusters of real cells, not individual ones. Rather, the value of $\phi$ is the expected fraction of proliferative cells, existing after treatment, whose resistance changes due to treatment. By varying this parameter, we can compare-within the limits of cellular resolution-the growth dynamics of a tumor to its mutational response to treatment. The term mutation is used here in a general sense, as it refers both to changes in gene sequences (genetic changes) and gene expression (epigenetic changes). The new $\gamma$-value is assigned randomly so that new strains can assume varying degrees of resistance, which may be quite different from those of parent strains. Also, the simulation does allow for back-mutations; if a treatment-resistant automaton cell is selected for a mutation, it may convert to a sensitive cell.

Overall, there are several important aspects of this treatment model. With the current set of growth parameters, the proliferation routine can simulate tumors that demonstrate realistic Gompertzian growth, while maintaining accurate ratios among growth and necrotic fractions (see paper I). The use of the Delaunay triangulation and the nutrient-dependent transitions among the cell types are likewise unique. By building our chemotherapy model upon this proliferation routine, we incorporate these considerations and study brain tumors from a global perspective of both growth dynamics and clinical response.

The automaton nature of our model is itself advantageous. Foremost, automaton simulations do not ignore a tumor's microscopic composition while studying macroscopic behavior. Moreover, since it relies upon automaton cells, our model is well suited to investigate tumors with multiple sub-populations. The competitive interactions among strains are accounted for implicitly within the algorithm. As a result, while the equations that govern the simulations are quite simple, we can nonetheless account for numerous types of heterogeneities. The three-dimensional, isotropic nature of the cells also reflects the importance of connectivity and topology in a competitively-interacting milieu. It is our hope that this mathematically straightforward, yet thorough, model can be appreciated by theoreticians and clinicians alike.

Before discussing the data, it is important to describe a method of data analysis employed throughout this investigation. As previously mentioned, the simulations rely upon pulsed therapy in which the effects of treatment are assumed to be instantaneous. This method produces discrete (discontinuous) drops in tumor volume at the moments of treatment. Throughout this work, however, time is commonly analyzed as a function of tumor volume (as depicted in the survival time data of Figs. 4, 6 and 8). Consequently, the discontinuous data are converted to continuous growth curves. The discontinuous plot in Fig. 2 depicts tumor volume versus time for a simulated tumor. The reader should note the volumetric increases that occur during proliferation and volumetric drops that occur at each four-week instance of treatment. At each of these times, we calculate the average of the pre- and posttreatment tumor volumes; splines fits are performed on

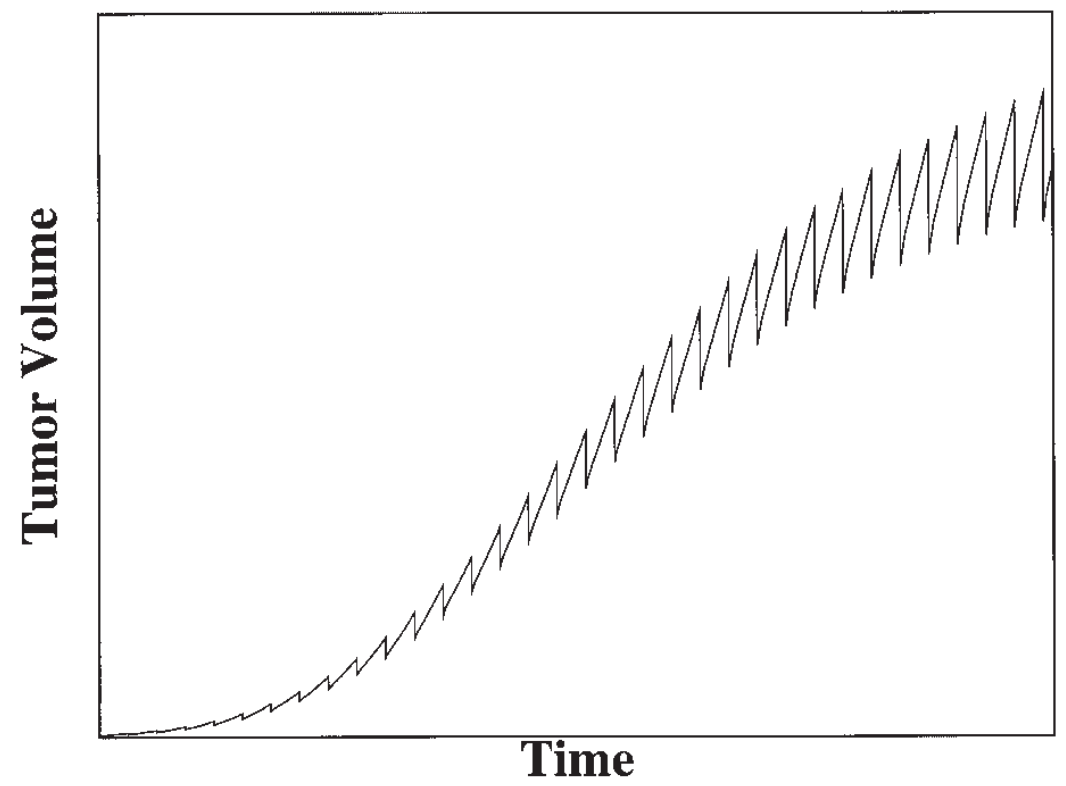

FIGURE 2 The data-fitting method. The discontinuous volume-versus-time plots of tumor growth produced by the simulation are converted to continuous curves. The procedure is described in the body of the text. 
these data points to generate smooth, continuous curves. The final, fitted data is overlaid on the raw plot in Fig. 2.

The programming for this investigation was written in the $\mathrm{C}++$ programming language on a UNIX operating platform. Each simulation was run in parallel on nine nodes of an IBM SP-2 processor. The work here encompassed approximately six weeks of computational processing time.

\section{RESULTS}

Our investigation consists of three individual case studies, each of which is presented in this section. In Case 1, the growth dynamics of monoclonal tumors are studied to determine how tumor behavior is affected by the treatment parameters $\gamma$ and $\epsilon$. Case 2 builds upon this information, analyzing the behavior of two-strain tumors. Here, a secondary treatment-resistant strain exists alongside a primary treatment-sensitive strain. We introduce the secondary sub-population at the onset of each simulation, initializing it in different spatial arrangements and at several (small) relative volumes. In both Cases 1 and 2, no additional sub-populations arise in the tumors once the simulation has begun (i.e. $\phi=0$ ). In Case 3 , however, we investigate tumors that are capable of undergoing resistance mutations in response to each round of treatment $(\phi>0)$. In these simulations, the growth and morphology of the tumors are analyzed in relation to the fraction of mutating cells.

\section{Case 1: Monoclonal Tumors}

The preliminary goal of this investigation is to examine the development of monoclonal tumors under the effects of treatment. Although real-life GBM tumors are multiclonal, it is important to study these simplified systems first to determine how tumor behavior is effected by the values of $\gamma$ and $\epsilon$ The growth behavior of these tumors is also compared to survival time data from clinical studies of GBM treatment. For all simulations of Case 1, $\phi=0$.

Fixing the value of $\epsilon$ at 0 , we first investigate the growth of monoclonal tumors as a function of their treatment sensitivity $(\gamma$-value). Figure 3 presents spline-fitted volume-versus-time plots for tumors with $\epsilon=0$ and $\gamma=$ $0.55,0.65,0.75,0.85$ and 0.95 . $\gamma=0.55$ corresponds to the most treatment-resistant tumor, while $\gamma=0.95$ corresponds to the most treatment-sensitive tumor. The scale of the vertical axis reflects biologically plausible volumes of GBM tumors. The reader should note that the spacing between the five growth curves grows as $\gamma$ increases: the difference between the $\gamma=0.85$ and 0.95 curves is greater than that between the $\gamma=0.55$ and 0.65 curves. For $\gamma$-values below 0.55 (not shown), there is little significant difference among curves' positions. These data suggest that-for an already resistant tumor-a small change in treatment sensitivity does not greatly affect

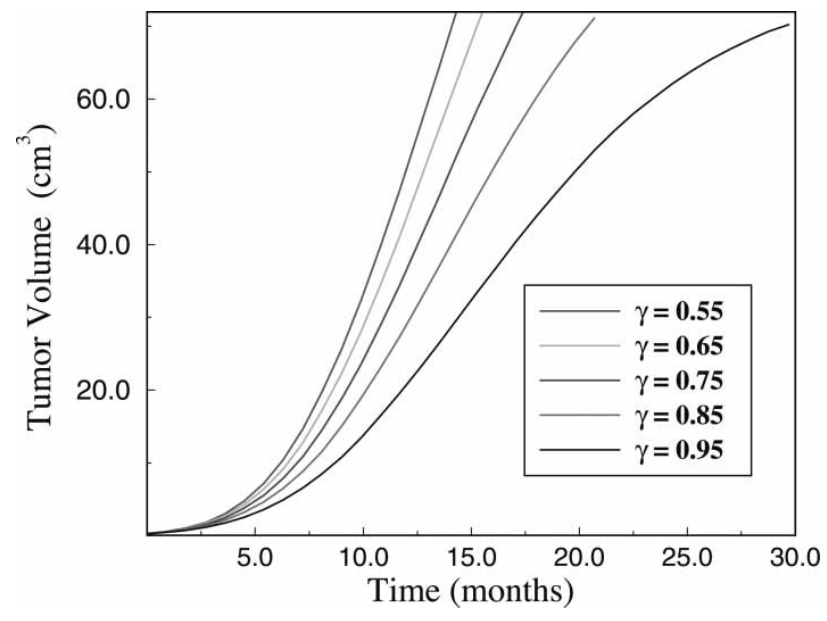

FIGURE 3 Representative tumor growth. The figure depicts five fitted growth curves, for which $\gamma=0.55-0.95$ and $\varepsilon=0$.

tumor growth. Conversely, if a tumor is treatmentsensitive, such a change would alter more appreciably its treatment response. At large times, the curves in Fig. 3 display asymptotic volumetric behavior; this is most evident in the $\gamma=0.85$ and 0.95 cases. In terms of gross morphological features, these monoclonal tumors develop with a nearly spherical geometry. They appear much like the monoclonal tumors that have been observed previously outside the context of treatment (see paper I).

For each tumor, a survival time is assigned based upon its growth curve. In paper I, we defined $65.5 \mathrm{~cm}^{3}$ as a lethal tumor volume based upon in vivo data. This standard was used to designate a time-of-death and to compare the simulation data to that of medical test cases. The $65.5 \mathrm{~cm}^{3}$ standard is likewise maintained here. Throughout the remainder of this report, survival time refers to the time at which a given tumor's spline-fitted growth curve is at the lethal volume.

Figure 4 indicates the survival times associated with several monoclonal tumor-systems, in which both $\gamma$ and $\epsilon$ are varied. The vertical axis depicts survival time in months, and the horizontal axis depicts the tumor's $\epsilon$-value. Each of the five curves (level sets) portrays a particular $\gamma$-value. The value of $\gamma$ varies from 0.55 to 0.95 , while that of $\epsilon$ varies from 0 to $\sim 0.4$. Higher values of $\epsilon$ are not considered, reflecting the effects of phase resistance in the non-proliferative cells. Also, the reader should note that the data points in Fig. 4 do not contain error bars. A tumor with particular $\gamma$ - and $\epsilon$-values will display the same survival time from one simulation to the next.

The data in Fig. 4 indicate that - at lower values of $\gamma$ survival time does not vary significantly with $\epsilon$. The level sets for $\gamma=0.55,0.65$ and 0.75 are nearly flat. By contrast, for the $\gamma=0.95$ case, the level set is relatively steep and only three data points are shown $(\epsilon=0.0,0.05$ and 0.1 ). When $\gamma=0.95$ and $\epsilon>0.1$, the tumors do not grow to a lethal volume, instead reaching their volumetric asymptote at lower sizes. In a situation tantamount to 


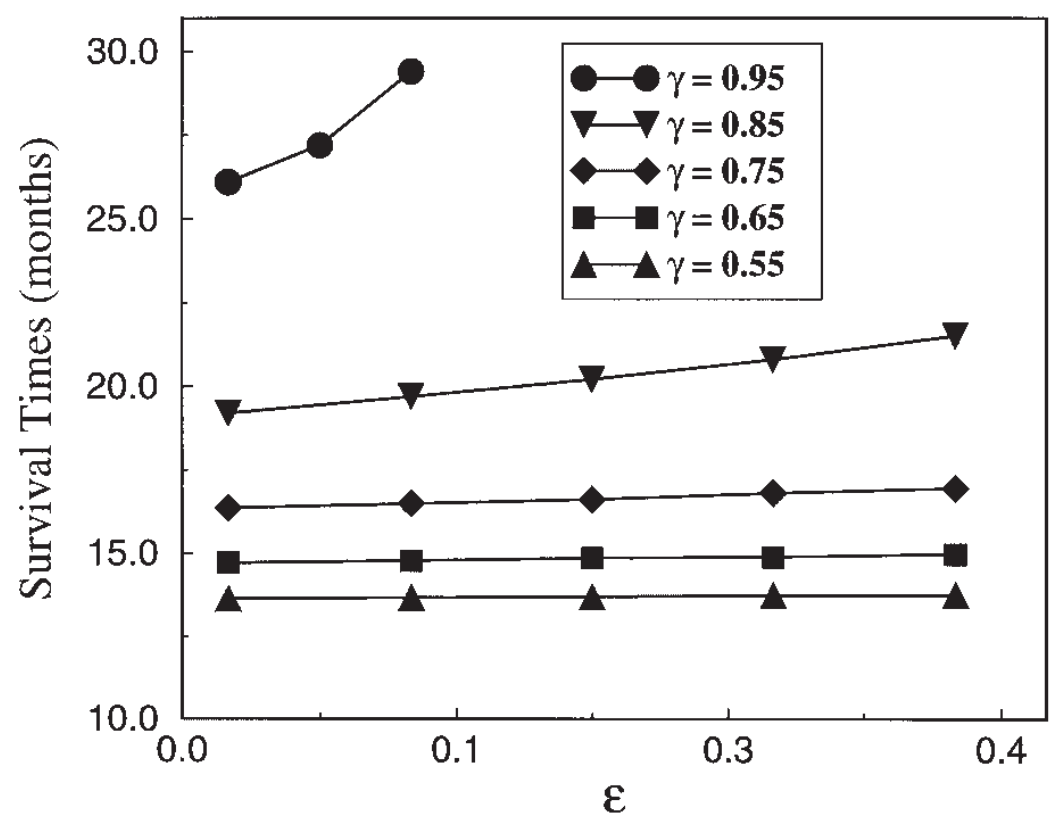

FIGURE 4 Survival times with variable $\gamma, \epsilon$. Depicted here are the associated survival times for tumors with various $\gamma$ and $\epsilon$ values.

tumor death, they convert to a mass of necrotic cells with subsequent rounds of treatment. In general, the tumors represented in Fig. 4 display a complimentary relationship between the value of $\gamma$ and the importance of $\epsilon$ : as a tumor's $\gamma$-value becomes larger, its $\epsilon$-value has a more significant impact on tumor growth. As one might expect in real life, the response of the actively-dividing cells in the simulation is of paramount importance. If the proliferative cells are highly resistant, the non-proliferative response is of little consequence for patient prognosis.

In addition to these growth trends, we are also interested in comparing the results of the treatment model to clinical statistics on GBM survival times. Paper I demonstrated that the proliferation routine is capable of generating realistic growth outside the context of treatment. In that same regard, we now wish to determine if the modified algorithm can maintain realistic growth with treatment added. We want to ensure that the range of observed survival times is qualitatively reasonable. We must emphasize, however, that our theoretical data should not be used to analyze quantitatively real-life GBM tumors. For example, we are not suggesting that one can crossreference the values in Fig. 4 to a patient's survival time to obtain the exact level of resistance of that patient's tumor.

Over the past decade, several studies have described the survival times of GBM patients undergoing aggressive treatment. A study first published in Holland (2000) examines treatment efficacy in $184 \mathrm{GBM}$ patients who underwent surgery ( $>95 \%$ resection), radiation therapy, and chemotherapy. The median survival time among these patients was roughly 1 year, and roughly $20 \%$ survived over 2 years. A study of grades III (anaplastic astrocytoma) and IV (GBM) brain tumors from Salcman et al. (1994) divided patients into age brackets of $>40$ years and $\leq 40$ years. The median survival time of older patients with aggressive treatment was 16 months, while that of the younger patients was 24 months. As Fig. 4 indicates, the wide range of survival times that the simulation can produce is medically plausible.

We should note here that the survival time associated with a totally resistant tumor $(\gamma=0, \epsilon=0)$ is roughly 11 months. With its current method of surgery (99\% resection), its regiment of treatment (every four weeks), and its definition of lethal volume $\left(65.5 \mathrm{~cm}^{3}\right)$, the simulation cannot produce very low survival times on the order of several months. A $\gamma=0, \epsilon=0$ tumor corresponds to a treatment scenario in which only surgery occurs without subsequent chemotherapy or radiation treatment. In a study by Jelsma and Bucy (1967), roughly $25 \%$ of surgery-only patients survived a year following resection. The simulated survival time tumor falls within a reasonable range, but it is on the high end. It is important to mention, though, that most surgery-only clinical data is relatively old, published before the advent of MRI scanning technology.

\section{Case 2: Heterogeneous Tumors, Two Strains}

We next focus upon heterogeneous tumors, in which subpopulations differ in terms of their response to treatment. We look at two-strain tumors, in particular. The tumors are initialized with a dominant sensitive strain and a secondary resistant strain. As the simulations run, tumor growth can be tracked as the sub-populations evolve in a competitive context. As in Case $1, \phi=0$ in Case 2.

Before performing any analysis, we must address the issue of how the resistant strain is to be initialized within the sensitive one: should the resistant cells be localized to one specific region of the tumor, or should they be distributed at various locations? It is important to recall 
that the initial tumors in these simulations represent postoperative tumors. Even if a resistant strain is confined to a particular spatial location in a tumor before surgery, it may not be after surgery.

In order to account for any surgical disruption of tumorous tissue (e.g. the collapse/rearrangement of an initial margin of post-operative cells), we model two extreme possibilities for the arrangement of the secondary strain. We investigate systems in which the resistant cells are (1) localized to a specific spatial location in the tumor and (2) scattered uniformly throughout the tumor. The first scenario is referred to here as the localized resistance scenario, and it assumes that surgery does not significantly alter the spatial arrangement of pre-surgical strains. The second scenario is referred to as the scattered resistance scenario, and it assumes that the spatial arrangement of the cells left behind is unrelated to their original positions. The reader should note that the scattered case could also be employed to analyze the behavior of a tumor in which several small, resistant strains had arisen prior to surgery. The images of Fig. 5 depict representative tumors of each scenario at the onset of a simulation.

By investigating the different spatial arrangements, we are probing a fundamental issue with regard to the microscopic interactions between the two sub-populations. Inter-strain competition occurs only where resistant and sensitive cells interface one another (i.e. share a common automaton surface). In the localized scenario, this interface only exists at the border between the two strains. Conversely, in the scattered scenario, all resistant automaton cells are surrounded (or nearly surrounded) by sensitive cells. As a result, the total interfacial area per number of resistant cells (IA/RC) is much higher in scattered scenario compared to the localized one. Overall, we will determine whether a resistant sub-population is more strongly favored by a higher or a lower IA/RC.

To address this issue, we determine for both scenarios how tumor growth varies with the size and level of resistance of the secondary population. In all simulations in this case, the treatment parameters of the sensitive population are fixed at $\gamma=0.95, \epsilon=0.05$. (a)

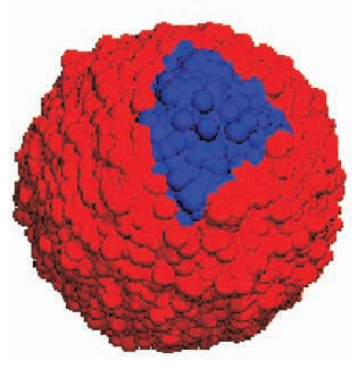

(b)

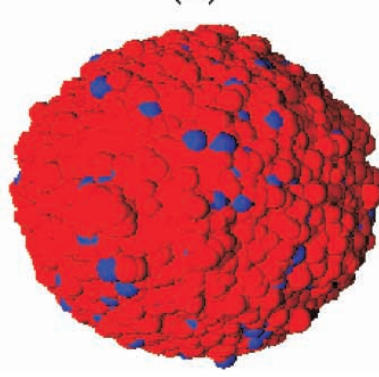

FIGURE 5 Initial images of two-strain tumors. The resistant subpopulation is localized in (a) and scattered in (b). The blue cells of each tumor belong to the resistant sub-population, while the red ones belong to the sensitive sub-population.
For a monoclonal tumor, these parameters would correspond to a survival time of 27 months. The secondary strain is initialized at a variety of $\gamma$-values (all less than $0.95)$, and at several relative volumes. For a description of how this process is executed for both scenarios within the computational programming, the reader is referred to the Appendix, part B. In these simulations, the value of $\epsilon$ is held constant at 0.05 for all strains. We define the term $\beta$ as the volumetric fraction of living cells (proliferative and non-proliferative) that belong to the resistant strain.

Figure 6 shows how a tumor's survival time varies with the $\gamma$ - and initial $\beta$-values $\left(\beta_{0}\right)$ of the resistant strain. Figure 6 a presents the data for the localized resistance scenario, and Fig. $6 \mathrm{~b}$ does so for the scattered resistance scenario. For both graphs, the horizontal axis specifies the $\gamma$-value of the resistant strain, while each of the level sets indicates $\beta_{0}$. For the values of $\beta_{0}$ considered here, we observed no examples of the resistant sub-population being overwhelmed by the sensitive one and dying out. As in the monoclonal case, there is no significant variation in survival time from one simulation to the next for a particular two-strain tumor.

While the overall shape of the level sets is similar in Fig. 6a,b, the survival times associated with the scattered tumors are lower than their localized counterparts. In the scattered simulations, the resistant strains more significantly decrease the survival times from the monoclonal, sensitive level of 27 months. This reveals that scattered resistant strains are able to compete more effectively than localized ones. Figure 7 further exemplifies the observation, depicting $\beta$ as a function of time for representative simulations. The figure compares the localized and scattered scenarios for two particular combinations of $\beta_{0}$ and $\gamma$ (of the resistant strain): $\beta_{0}=0.05, \gamma=0.2$ and $\beta_{0}=0.05, \gamma=0.8$. In both graphs, the scattered resistant strains overwhelm the primary strains throughout the course of treatment. By contrast, in the localized simulations, a significant fraction of sensitive cells remain in the tumors even once they have reached a lethal volume. In the localized simulation where $\beta_{0}=0.05$, $\gamma=0.8$, the secondary strain never comprises a majority of the tumor.

Due to this behavior, the three-dimensional development of the tumors may differ in the localized and scattered scenarios. The tumor image in Fig. 8a portrays the scattered resistance tumor of Fig. 7a $\left(\beta_{0}=0.05, \gamma=\right.$ 0.2 ) at the end of the simulation. It appears much like a tumor that had been initialized as a single, resistant strain. Figure $8 \mathrm{~b}$, on the other hand, portrays the localized resistance tumor of Fig. $7 \mathrm{~b}\left(\beta_{0}=0.05, \gamma=0.8\right)$. The two sub-populations appear as distinct regions of the fullydeveloped tumor. In the case that a localized resistant strain is able to overwhelm the sensitive strain entirely (i.e. for low $\gamma$-values and high $\beta_{0}$-values), the fully developed tumor will appear like the spherical image in Fig. 8a.

Overall, the results from Figs. 6 and 7 indicate that a high IA/RC more strongly favors the proliferation of a resistant sub-population. A lower IA/RC allows 
(a)

\section{Localized Secondary Strain}

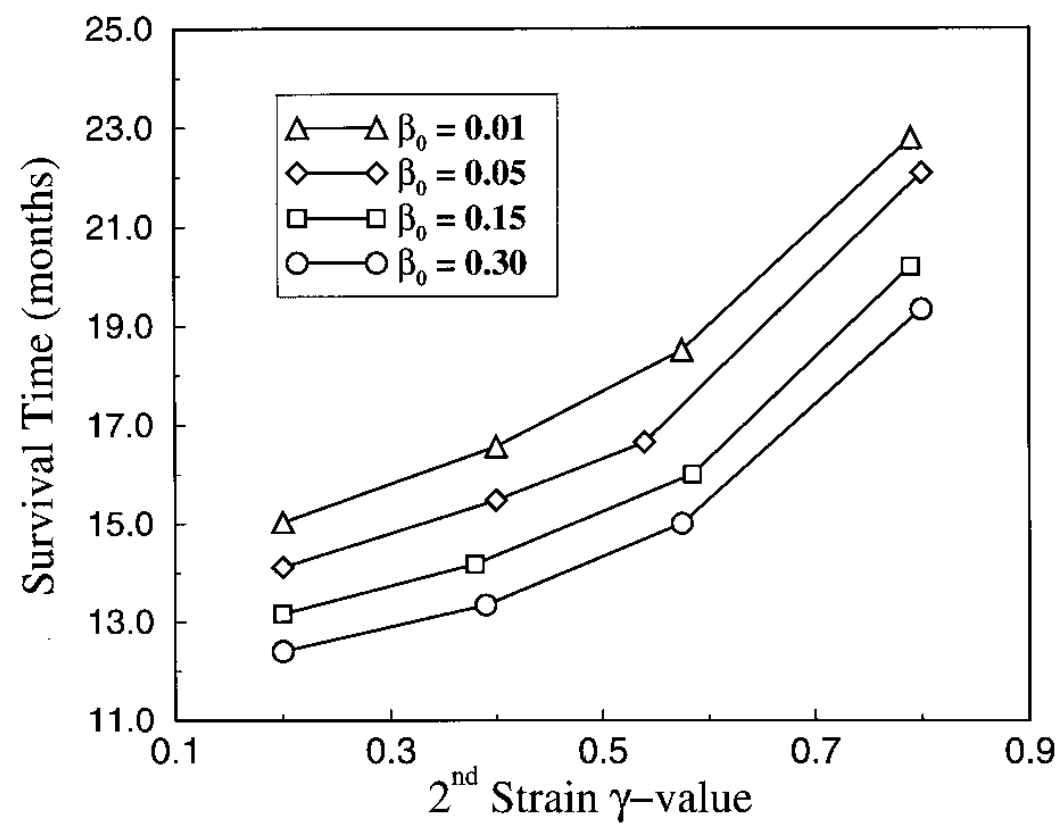

(b)

Scattered Secondary Strain

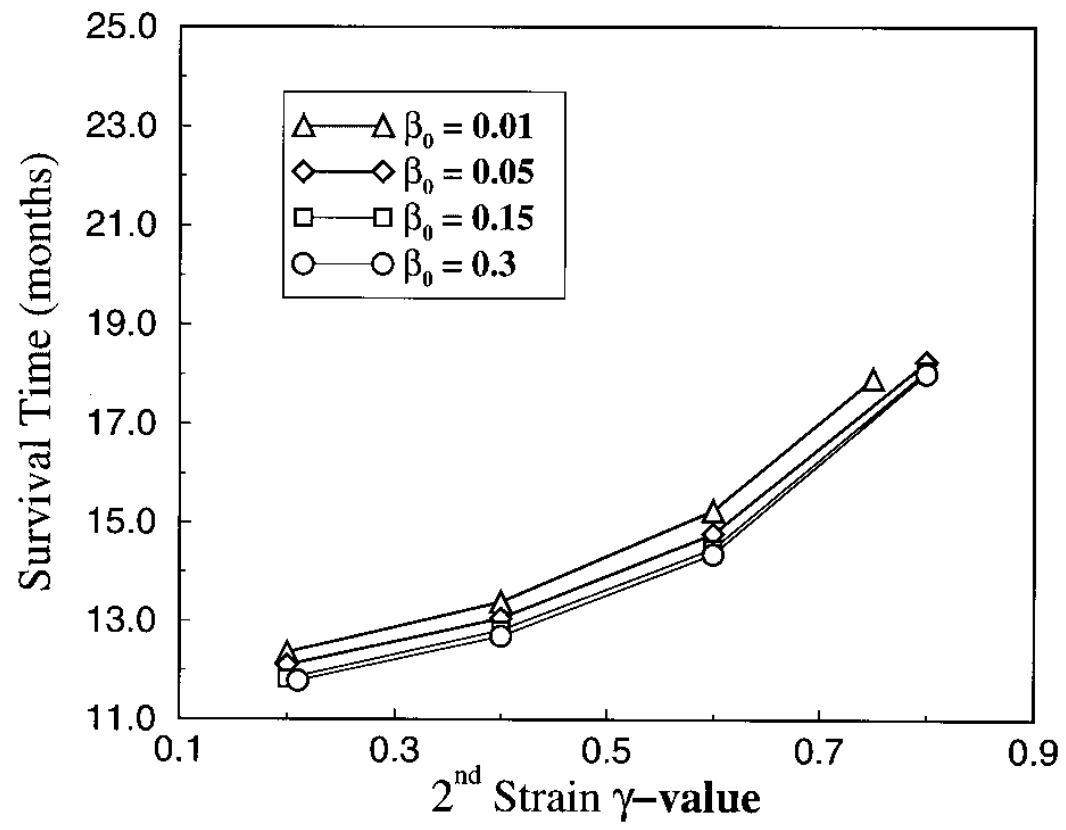

FIGURE 6 Survival times associated with various two-strain tumors. Each figure demonstrates how the survival time associated with a two-strain tumor varies with the size and severity of its resistant sub-population. (a) Depicts the data for the localized scenario, while (b) depicts the data for the scattered scenario.

the sensitive population to compete more effectively. This instance is not the first time that we have witnessed such a phenomenon in our automaton investigations of tumor growth. In paper II, we focused upon the dynamics of two-strain tumors in which each strain possessed a different growth-rate. As part of that study, we introduced localized, mutant sub-populations at very low $\beta_{0}$-values. Their probability of emergence was measured as a function of the new $p_{0}$-value $\left(p_{1}\right)$. We observed thateven for mutant sub-populations at a competitive disadvantage $\left(p_{1}<p_{0}\right)$-there existed a non-zero probability of emergence. Occasionally, a secondary strain with a lower growth-rate was able to proliferate significantly beyond its initial $\beta$-value. This behavior was again attributable to localization. Since the two strains shared a relatively low interfacial area, the automaton 
(a)

$\beta_{0}=0.05 ; \gamma=0.2$

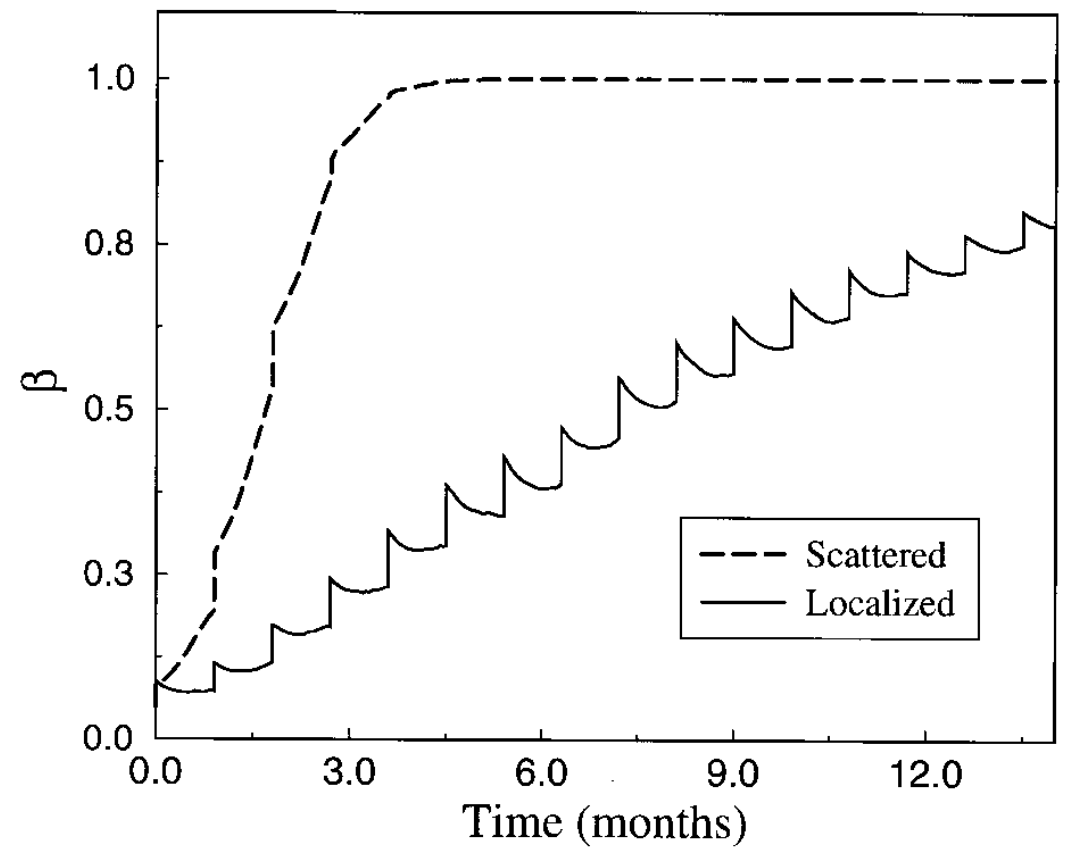

(b)

$\beta_{0}=0.05 ; \gamma=0.8$

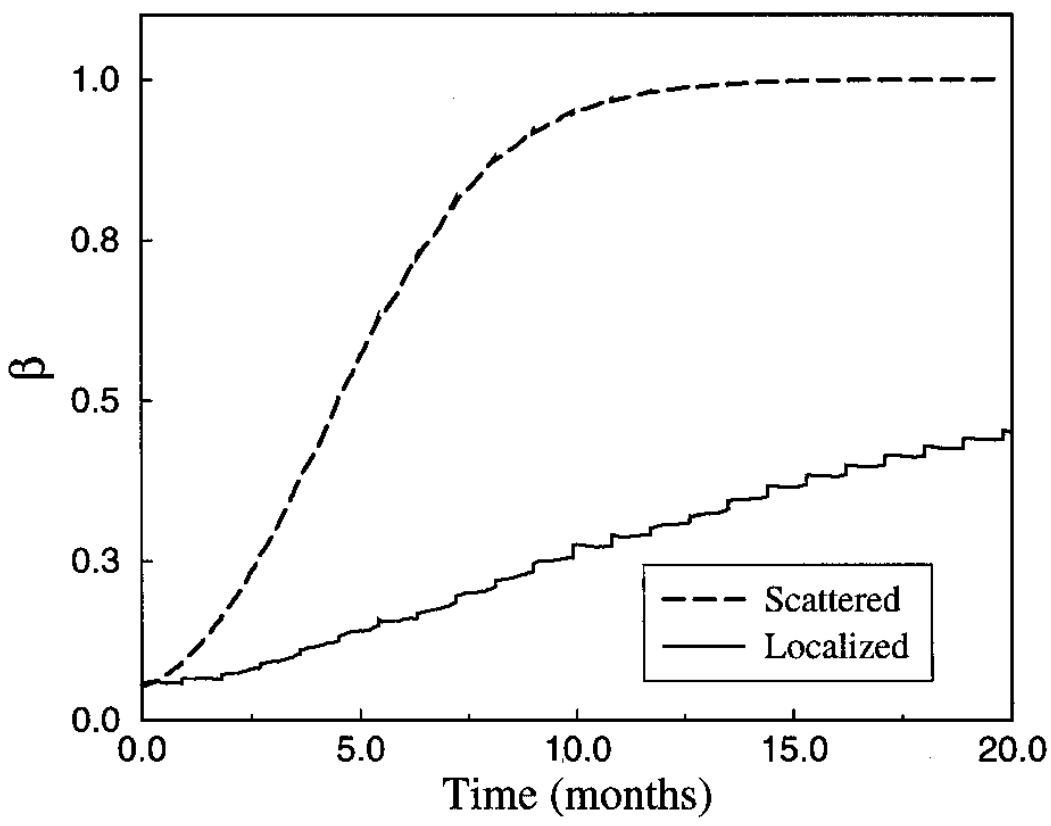

FIGURE $7 \beta$ versus time for two-strain tumors: localized versus scattered scenarios. These figures depict the $\beta$-value of a resistant strain as a function of time for the localized and scattered resistance scenarios. For (a), $\beta_{0}=0.05$, while the $\gamma$-value of the resistant strain equals 0.2 ( $\gamma$ of the sensitive strain equals 0.95). The dashed line depicts the ensuing $\beta$-values of a representative scattered-resistance tumor, while the solid line depicts those of a localizedresistance tumor. In (b), $\beta_{0}=0.05$ and $\gamma=0.8$.

cells at the interior of the mutant strain had no risk of being overwhelmed.

Compared to the current investigation, the selection pressure in paper II was different (growth-rate competition versus treatment effects). Moreover, the roles of the primary and secondary strains are reversed in the above example: the primary strain possessed a competitive advantage over the secondary strain. Nevertheless, both paper II and the current study indicate the same principle. The proliferative ability of a tumorous strain with a competitive advantage varies directly with its interfacial area per cell. 

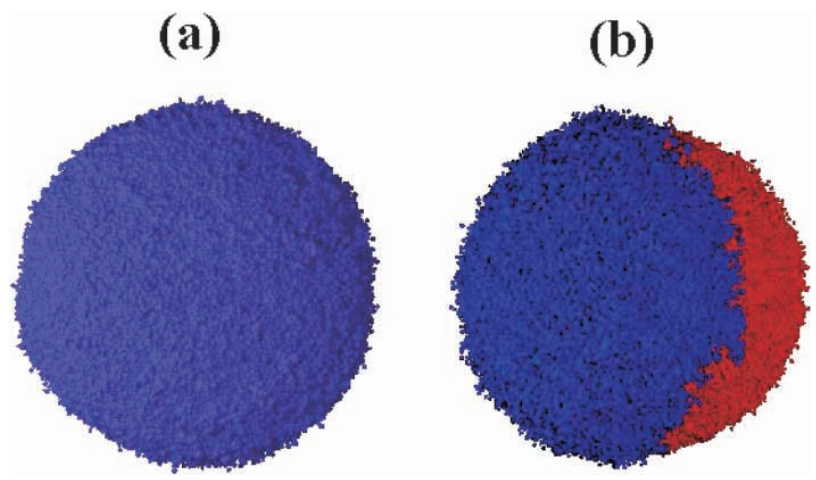

FIGURE 8 Final images of two-strain tumors. In (a), a scattered resistant strain has completely overwhelmed a sensitive strain. The tumor appears monoclonal and maintains a nearly spherical geometry. In (b), by contrast, a localized resistant strain has not completely overwhelmed a sensitive strain. The two sub-populations of the fully-developed tumor appear as distinct regions.

In addition to this observation, it is important to discuss one other trend in the survival time data of Fig. 6. In the scattered resistance scenario, the survival times show a very weak dependence on $\beta_{0}$ compared to the localized scenario. This observation goes hand-in-hand with the above discussion, as the survival time differences between the two scenarios are much more pronounced at higher $\beta_{0}$-values. In total, the data indicate that the total number of resistant cells in a heterogeneous tumor is not as important an indicator of patient prognosis when the cells are not localized to a particular region.

One can again interpret this trend by considering the microscopic interactions between the resistant and sensitive populations. In a typical simulation, a tumor's proliferative rim maintains a thickness of roughly three automaton cells. As a result, with the scattered $\beta_{0}=0.01$ case, approximately $30 \%$ of a tumor's sensitive, proliferative cells are in competition with a resistant cell immediately following initialization. After the first instance of treatment, only a few time-steps of resistantcell growth are needed for this fraction to reach $100 \%$. So for even the smallest secondary population under consideration, all sensitive cells are rapidly in competition with resistant ones. Therefore, additional increases in $\beta_{0}$ are of little consequence for tumor growth. With the localized resistant strains, the lower IA/RC precludes these rapid interactions involving all sensitive cells. In general, the dynamics of interacting spheres (or polyhedra, here) is a problem of much interest to materials science and statistical mechanics. The reader is referred to Torquato $(1984 ; 2002)$ for a description of the "cherrypit" model of interacting/overlapping spheres, on which the brief analysis above is based.

\section{Case 3: Heterogeneous Tumors, Induced Mutations}

In our third case study, we investigate the behavior of tumors that are able to develop resistance throughout the course of treatment. Unlike Case 2, the tumors in Case 3 begin the simulations as a single strain. Here, however, treatment can induce the appearance of mutant strains $(\phi>0)$. The goal of Case 3 is to relate the growth dynamics of brain tumors to their mutational response to treatment. Specifically, we are interested in the behavior of tumors that begin the simulations as treatment-sensitive but develop resistance over time. The tumors in Case 3 are all initialized monoclonally with $\gamma=0.95$ and $\epsilon=0.05$.

At first, the tumors in Case 3 will develop like treatment-sensitive, monoclonal tumors; growth will then accelerate as resistant cells begin to dominate. This corresponds to a case of acquired resistance via induced (genetic and epigenetic) mutations. Overall, the tumor dynamics here are more variable than in Cases 1 and 2 . When a new strain appears, it begins as a single automaton cell. Unlike case 2, not all new strains will be able to proliferate to an appreciable extent. Some are overwhelmed by the parent strain from which they arise. In addition, we do not limit the number of distinct subpopulations that can arise throughout the course of a simulation (or the time at which they arise).

Again, we are interested in the survival times associated with various tumor-systems. The mean survival time of the tumors was determined as a function of $\phi$. Figure 9 plots this data; at least 50 simulations were performed for each value of $\phi$. Unlike Figs. 4 and 6, error bars are included in Fig. 9-there is significant variability in tumor growth at a single value of $\phi$. From $\phi=10^{-5}$ to $\phi=10^{-2}$, the survival times vary nearly logarithmically with $\phi$. When $\phi=10^{-5}$, the mean time is near 27 months, as most tumors remain monoclonal (or nearly monoclonal) with $\gamma=0.95, \epsilon=0.05$. As $\phi$ increases, resistant strains appear more commonly and survival times fall (see the discussion on tumor morphologies to follow).

The survival times in Fig. 9 must show asymptotic behavior, though, at either extreme of the horizontal axis. Independent of the value of $\phi$, survival time cannot exceed 27 months, nor can it fall lower than 11 months. The latter time corresponds to that of a totally resistant $(\gamma=0, \epsilon=$ 0.05 ) tumor. These two times are indicated in Fig. 9 with dashed lines. The reader should note that a $\phi$-value as low $10^{-4}$ can decrease survival time by more than six months. These times should not be interpreted as quantitative measures of the behavior of actual GBM tumors, as the treatment sensitivity of the parental strain is predefined. The data in Fig. 9 are again intended only to be a more qualitative picture that links a particular tumor's growth behavior to its mutational response.

\footnotetext{
${ }^{\star}$ With this initial $\gamma$-value, nearly every mutant strain that arises from the initial population will posses a lower $\gamma$-value. We are not suggesting that all induced mutations must possess increased resistance. This fact here merely stems from the initial sensitive tumor under consideration.
} 


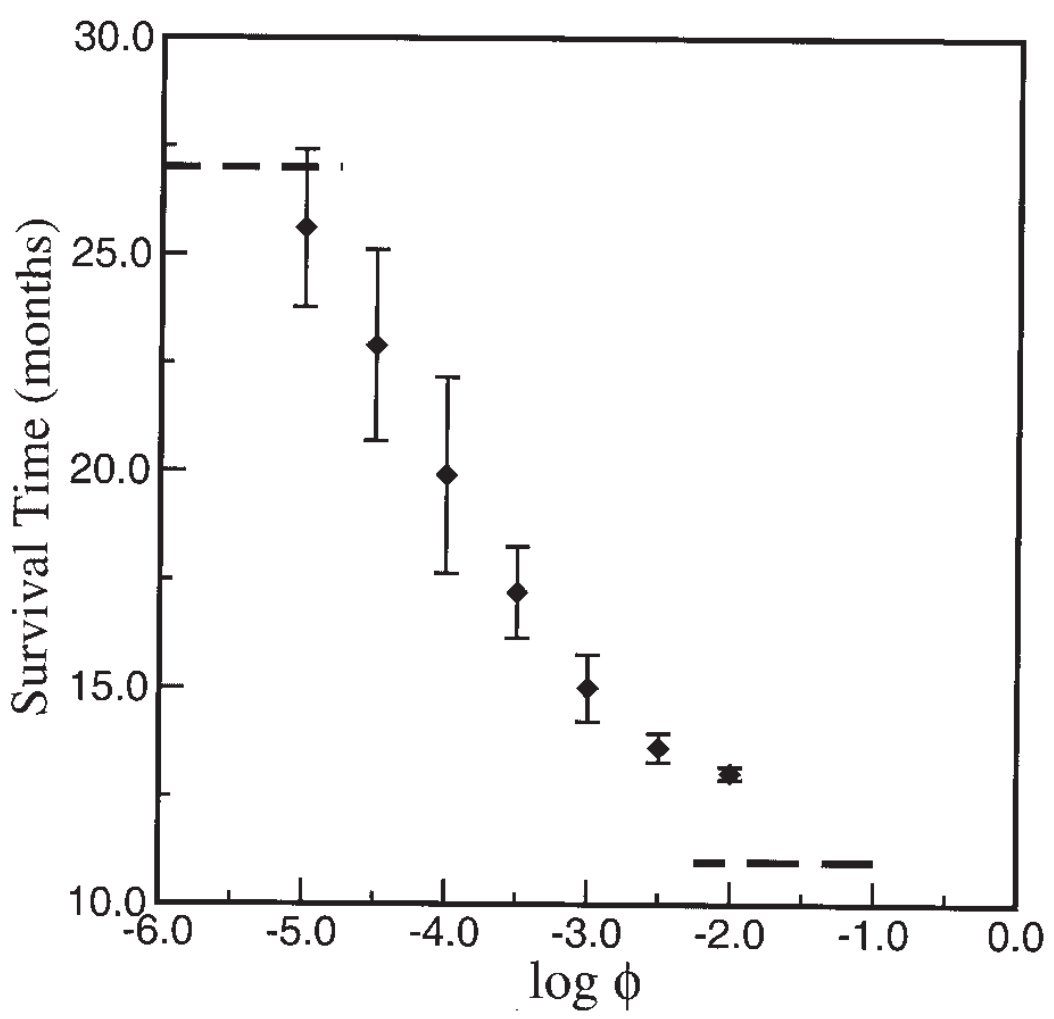

FIGURE 9 Survival times associated with continuously mutating tumors. This figure depicts mean survival time as a function of the log of $\phi$, the expected fraction of tumor cells that mutate at each instance of treatment $(n=50)$.

One of the more intriguing observations in Case 3 involves the gross morphology of the mutating tumors. Their three-dimensional geometries exhibit an interesting dependence on the value of $\phi$. Figure 10 presents representative images of the fully-developed tumors for $\phi=10^{-5}, 10^{-4}, 10^{-3}$ and $10^{-2}$. For $\phi=10^{-5}$ (Fig. 10a), some tumors develop a secondary strain while others do not. The tumors that remain monoclonal maintain their spherical geometry. When a resistant sub-population does develop, it appears as a lobe on the parental tumor. As $\phi$ increases above $10^{-5}$, resistant sub-populations consistently arise from the parental strain. The images in Fig. 10b,c depict these tumors, whose geometries consistently deviate from an ideal sphere. These tumors are multi-lobed in appearance, and the original strain is commonly over-whelmed. However, when $\phi$ grows even greater, the geometric trend reverses. The $\phi=10^{-2}$ tumors in Fig. 10d again appear more spherical, despite the fact that they experience the greatest fraction of mutations per treatment event. These images suggest that extreme mutational responses can lead to similar macroscopic geometries. Non-spherical geometries result from intermediate $\phi$-values. Although Fig. 10 only depicts several representative images for each $\phi$-value, the geometric trend holds true for all simulations. The $\phi=$ $10^{-2.5}$ and $10^{-2}$ tumors were markedly more spherical in appearance than the $\phi=10^{-3}$ to $10^{-4}$ tumors.

This behavior may be appreciated in light of the observations of tumor behavior in the first two case studies. When $\phi$ is high, a large number of automaton mutations occur with each round of treatment. Similar to the scattered resistance scenario of Case 2, the mutant cells are distributed uniformly over the tumor's surface. As resistant strains begin to develop, they do so throughout the tumor. Moreover, for a large range of $\gamma$-values, the mutant strains will develop similarly: as was observed in Case 1, a strain's volumetric growth does not vary as significantly with $\gamma$ when $\gamma$ is lower $(\gamma=0$ to $\sim 0.5$ ). Ultimately, it is these resistant strains that come to dominate throughout the course of a simulation. So even though these fully-developed tumors will possess a number of sub-populations, they can maintain a spherical geometry. For intermediate $\phi$-values, by contrast, fewer resistant cells appear per treatment instance and their spatial distribution is not as uniform. Rather, the induced sub-populations will exist at a few specific points on the tumor surface. This behavior is similar to the localized resistance scenario of Case 2, except in this case new strain begins at a very low $\beta$-value (i.e. a single automaton cell). Consequently, the resistant cells develop as lobes on the parental strain.

\section{DISCUSSION AND CONCLUSIONS}

In this work, we describe an automaton model of brain tumor treatment extended from a previously-designed proliferation model. The simulation treats automaton 


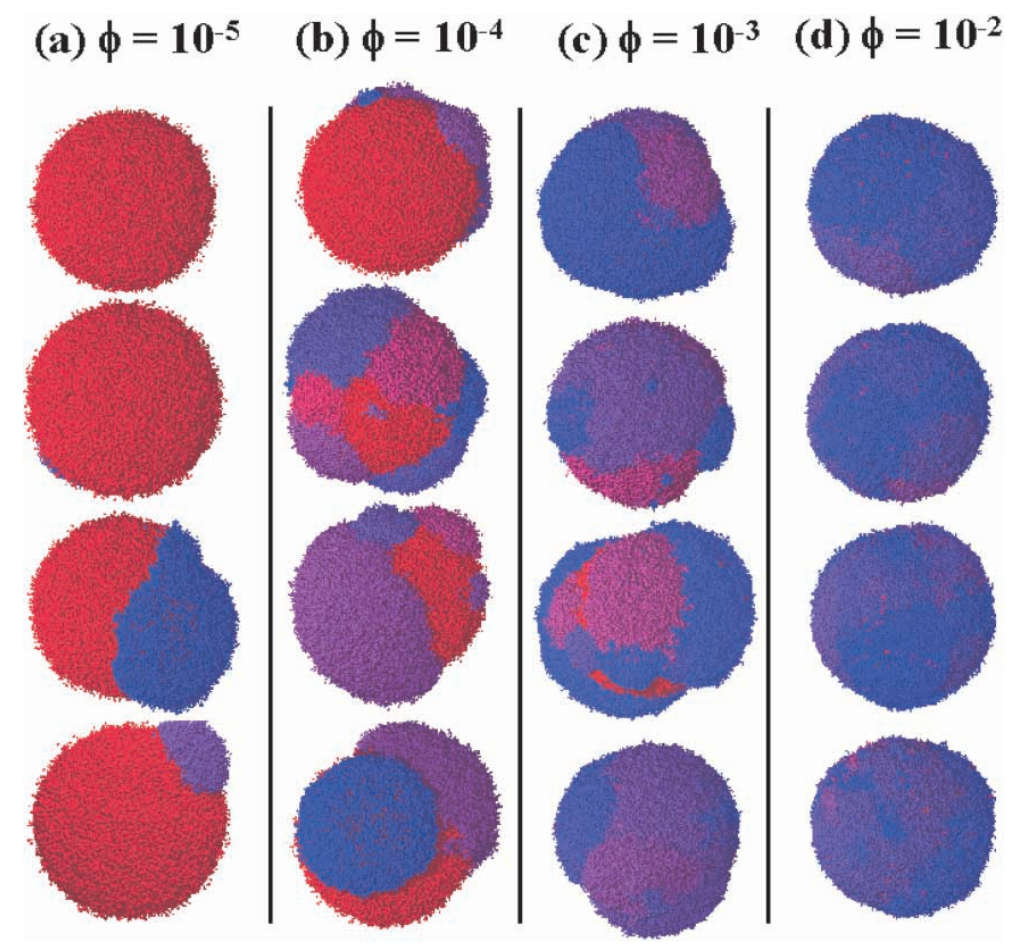

FIGURE 10 Images of continuously mutating tumors. We present representative images of tumors with the following $\phi$-values: (a) $\phi=10^{-5}$, (b) $\phi=10^{-4}$, (c) $\phi=10^{-3}$ and (d) $\phi=10^{-2}$. The distinct clonal sub-populations in each tumor are represented with a different color, ranging from red (highest $\gamma$-values) to violet (lowest $\gamma$-values). All tumors here are fully-developed.

events such as cellular divisions, mutations, and treatment-induced deaths probabilistically. We investigate three different tumor-systems under the effects of treatment: monoclonal tumors, two-strain tumors with resistant sub-populations, and multi-strain tumors with induced mutations. We present survival time data from each of these case studies, as well as important observations of tumor morphology.

Several of our findings are of particular significance, as they suggest trends in tumor behavior that may extend beyond a treatment/resistance context. The results from Case 2 regarding interfacial area are one such example. The data indicate that a resistant sub-population is able to dominate a tumor more rapidly and completely when it is not confined to a single location. In conjunction with previous results from paper II, this finding implies that a tumorous sub-population with a competitive advantage is most highly favored when the interfacial area among strains is maximized. In addition, the data from Case 2 suggest that the total volumetric fraction of non-localized strains is not as important a factor in tumor development. Although here we study only strains that differ in terms of treatment response, these principles of competition for scattered strains could extend to other scenarios. In reallife tumors, the behaviors might apply to clonal strains that differ in terms of other properties, such as nutritional requirements, toxicity responses, cellular adhesion, and angiogenic capacity. While our simulation does not investigate the mechanisms by which a single strain might come to exist in several locations, the effects of surgery may raise such behavior as a possibility.
The trends in tumor geometry observed in Case 3 could likewise be applicable outside the context of treatment. Both the highly mutating and the highly stable tumors develop with a more spherical geometry; tumors with an intermediate mutational response have a multi-lobed appearance. This morphological behavior might apply to mutations that occur continuously throughout tumor development, even without the mutagenic effects of treatment. GBM tumors are indeed characterized by their high genetic instability. For instance, one could consider random growth-rate mutations that occur throughout tumor growth. To examine this scenario, we have considered augmenting the proliferation routine to allow growth-rate $\left(p_{o}\right)$ mutations to occur with every cellular division. The geometries that these tumors adopt could be compared to the probability and severity of the mutations.

Granted, additional factors would have to be addressed to simulate the full complexity of dynamic mutations. These factors include the heterogeneous nature of surrounding brain tissue, as well as the possibility of a mutation affecting multiple cellular properties simultaneously. As mentioned above, strains may differ in a variety of ways, and a single genetic mutation might impact several traits. To investigate this issue in future treatment simulations, our model could allow induced mutations to alter both treatment parameters and growth parameters at once. In this initial investigation of tumor treatment-in which growth parameters are held constanta strain's relative resistance (i.e. its ability to survive and proliferate compared to other strains) depends solely on its $\gamma$ - and $\epsilon$-values. However, if growth parameters could also 
mutate, treatment-resistance would become a more complex phenomenon. Both a strain's response at instances of treatment and its growth-rate between treatments would impact its development. It would thus be intriguing to compare the fully-developed geometries of these tumors to the images of Fig. 10. Likewise, we could analyze whether mutations of a particular parameter are most strongly selected for throughout the course treatment.

Moreover, just as we could expand our model to account for more complex mutations, we could also augment it to simulate more complex treatment regiments. We do not currently consider the effects of crossresistance and multidrug resistance. Here, we analyze implicitly the effects of a single method of treatment prescribed at a time interval of one month. Modern treatment methods for GBM, however, can involve an array of drugs, specifically intended to counter the effects of cross-resistance. Future modeling work could, thus, focus on the effectiveness of various treatment regiments. In the simulation, this could be accomplished by sets of treatment parameters with correlation coefficients.

Overall, the research-extensions described in the preceding paragraphs could be accomplished through relatively straightforward modifications of the current algorithm. At the same time, there are a number of broader ways in which automaton models could advance in the future. For instance, a notable feature of GBM tumors is their ability to develop both through proliferative growth of a principle tumorous mass, as well as through invasive growth of migratory cells. While our model focuses on proliferative growth, future models could incorporate both modes of growth under a single automaton simulation. In particular, such a model could address the role of cellcell adhesion and its impact on invasion and treatment. Previously, automaton models have been utilized to investigate such invasive processes as vessel morphogenesis (Markus et al., 1999) and river network formation (Kim and Kim, 2001).

Another worthwhile goal of future brain tumor modeling would be to simulate directly the removal of a tumor and its effect on surrounding tissue. The simplified post-surgical tumors that begin each simulation do not consider the actual mechanics of tumor resection, nor do they allow for the effects of pre-surgical growth (e.g. previous angiogenesis). Granted, as discussed previously, we believe that our spherical method of initialization is suitable for the purposes of this study. Many of the observed growth trends deal with issues independent of the tumors' initial geometry. These trends include the low dependence of survival times on $\gamma$ when $\gamma$ is small, as well as the importance of interfacial area for inter-strain competition. Even the observations of tumor morphology in Case 3 can be appreciated in a generalized sense. Although these tumors are described in terms of their spherical appearance, this description fundamentally relates to the tendency of a tumor to shift its mass away from an initial location. This tendency may apply to tumors with initial geometries other than spheres. Nevertheless, it would be beneficial to model explicitly the process of resection and post-surgical tissue collapse within an automaton lattice.

Similarly, future models could calculate the continuous chemical gradients that chemotherapeutic agents form throughout the brain. A recent study by Patel et al. (2001) proposes a hybrid model that combines automaton cells with sets of differential equation to govern chemical diffusion. Their investigation does not consider tumor treatment, but it does provide valuable insights on how drug diffusion could be treated within an automaton lattice. The cells in their two-dimensional model are governed by a state vector that accounts for cell type and the local concentrations of nutrients and metabolic waste products. The behavior of a particular cell depends upon its neighbor cells and the chemical components of its state vector. A study of tumor metastasis from Anderson et al. (2000) also combines discrete cells with continuous concentrations. In a hybrid automaton model of tumor treatment, drug concentrations may be incorporated in a similar fashion; cellular responses to treatment could vary continuously with the local amount of drug.

In order to analyze macroscopic tumor treatment in this fashion, however, issues of computational processing speed must also be addressed. Since they consider chemical diffusion on length-scales of single biological cells, Patel et al. only analyze tumors of very small sizes. In fact, one of the greatest shortcomings of previous automaton models is their inability to investigate fullydeveloped tumors. Macroscopic brain tumors consist of too many individual cells for each one to be accounted for explicitly within a computational algorithm. We side step this issue in part by formulating automaton cells that represent clusters of real cells. Moreover-as described in paper I-automaton size increases at greater radial distances. These methods allow us to study brain tumors over their entire growth history, while maintaining high microscopic resolution at early times. Nevertheless, in an ideal situation, every automaton cell would be equal in volume and equivalent to one biological cell. This scenario would more readily allow for the explicit consideration of drug gradients. Similarly, the parameters that govern automaton behavior would map directly onto the behaviors of single tumor cells (not clusters). However, the resolution of simulations in the near term will continue to be limited by computational power.

In conclusion, this work represents a preliminary attempt to simulate the effects of tumor treatment with a novel automaton simulation. Our model indicates several behavioral trends for monoclonal, two-strain and dynamically mutating tumors. In general, we wish to demonstrate the ability of automaton models to address treatment issues. In the past, such simulations have not been highly utilized in this regard. As medical knowledge and computational power grow in the future, however, automaton models may be useful as part of an integrated approach to tumor treatment. 


\section{Acknowledgements}

This work has been supported in part by grants CA84509 and CA69246 from the National Institutes of Health. Calculations were carried out an IMB SP2, which was kindly donated by the IBM corporation (equipment grant to Princeton University for the Harvard-Princeton Tumor Modeling Project). We also thank Dr Thomas S. Deisboeck of Harvard Medical School for his insights on this project.

\section{References}

Anderson, A.R.A., Chaplain, M.A.J., Newman, E.L., Steele, R.J.C. and Thompson, A.M. (2000) "Mathematical modeling of tumor invasion and metastasis", Journal of Theoretical Medicine 2 , $129-154$.

Berkman, R.A., Clark, W.C., Saxena, A., Robertson, J.T., Oldfield, E.H. and Ali, I.U. (1992) "Clonal composition of glioblastoma multiforme", Journal of Neurosurgery 77, 432-437.

Birkhead, B.G., Rankin, E.M., Gallivan, S., Dones, L. and Rubens, R.D. (1987) "A mathematical model of the development of drug resistance to cancer chemotherapy", European Journal of Cancer and Clinical Oncology 23, 1421-1427.

Boldrini, J.L. (2000) "Therapy burden, drug resistance, and optimal treatment regimen for cancer chemotherapy", IMA Journal of Mathematics Applied in Medicine and Biology 17, 33-51.

Bredel, M. (2001) "Anticancer drug resistance in primary human brain tumors", Brain Research Reviews 35, 161-204.

Chadhary, P.M. and Roninson, I.B. (1993) "Induction of multidrug resistance in human cells by transient exposure to different chemotherapeutic drugs", Journal of the National Cancer Institute 85, 632-639.

Coldman, A.J. and Goldie, J.H. (1979) "A mathematical model for relating the drug sensitivity of tumors to their spontaneous mutation rate", Cancer Treatment Reports 63, 1727-1731.

Coldman, A.J. and Goldie, J.H. (1985) "Role of mathematical modeling in protocol formulation in cancer chemotherapy", Cancer Treatment Reports 69, 1041-1045.

Coldman, A.J. and Goldie, J.H. (1986) "A stochastic model for the origin and treatment of tumors containing drug-resistant cell", Bulletin of Mathematical Biology 48, 279-292.

Coons, S.W. and Johnson, P.C. (1993) "Regional heterogeneity in the DNA content of human gliomas", Cancer 72, 3052-3060.

Costa, M.I.S., Boldrini, J.L. and Bassanezi, R.C. (1995) "Drug kinetics and drug resistance in optimal chemotherapy", Mathematical Biosciences 125, 191-209.

Deisboeck, T.S., Berens, M.E., Kansal, A.R., Torquato, S., Stemmer-Rachamimov, A.O. and Chiocca, E.A. (2001) "Pattern of self-organization in tumor systems: complex growth dynamics in a novel brain tumor spheroid model", Cell Proliferation 34, $115-134$.

Düchting, W. and Vogelsaenger, T. (1985) "Recent progress in modeling and simulation of three-dimensional tumor growth and treatment", BioSystems 18, 79-91.

Endicott, L.C. and Ling, V. (1989) "The biochemistry of the Pglycoprotein-mediated multidrug resistance", Annual Reviews in Biochemistry 58, 137-171.

Gekeler, V., Beck, J., Noller, A., Wilisch, A., Frese, G., Neumann, M., Handgretinger, R., Ehninger, G., Probst, H. and Niethammer, D. (1994) "Drug-induced changes in the expression of mdr-associated genes-investigations on cultured cell lines and chemotherapeutically treated leukemias", Annals of Hematology 69, S19-S24.

German, U.A. (1996) "P-glycoprotein-a mediator of multidrug resistance in tumour cells", European Journal of Cancer $\mathbf{3 2 A}$, 927-944.

Gerweck, L.E., Kornblinth, P.L., Burlett, P., Wang, J. and Sweigert, S. (1977) "Radiation sensitivity of cultured glioblastoma cells", Radiology 125, 231-241.

Holland, E.C. (2000) "Glioblastoma multiforme: the terminator", Proceedings of the National Academy of Science 97, 6242-6244.

Jansson, B. and Revesz, I. (1974) "Analysis of the growth of tumor cell populations", Mathematical Biosciences 19, 131-154.
Jelsma, R. and Bucy, P.C. (1967) "Treatment of glioblastoma multiforme of brain", Journal of Neurosurgury 27, 388-400.

Kansal, A.R., Torquato, S., Chiocca, E.A. and Deisboeck, T.S. (2000a) "Emergence of a subpopulation in a computational model of tumor growth", Journal of Theoretical Biology 207, 431-441.

Kansal, A.R., Torquato, S., Harsh, IV, G.R., Chiocca, E.A. and Deisboeck, T.S. (2000b) "Simulated brain tumor growth dynamics using a three-dimensional cellular automaton", Journal of Theoretical Biology 203, 367-382.

Kayama, T., Yoshimoto, T., Fujimoto, S. and Sakurai, Y. (1991) "Intratumoral oxygen pressure in malignant brain tumors", Journal of Neurosurgury 74, 55-59.

Kim, H.J. and Kim, I.M. (2001) "Formation of river networks in heterogeneous surfaces", Journal of the Korean Physical Society 2, 497-499.

Kraus, M. and Wolf, B. (1993) "Emergence of self-organization in tumor cells-relevance for diagnosis and therapy", Tumor Biology 14, $338-353$.

Markus, M., Bohm, D. and Schmick, M. (1999) "Simulation of vessel morphogenesis using cellular automata", Mathematical Biosciences 156, 191-206.

Michelson, S. (1989) "Mathematical models for multidrug resistance and its reversal", Cytotechnology 12, 315-324.

Michelson, S., Ito, K., Tran, H.T. and Leith, J.T. (1989) "Stochastic models for sub-population emergence in heterogeneous tumors", Bulletin of Mathematical Biology 51, 731-747.

Okabe, A., Boots, B. and Sugihara, K. (1992) Spatial Tesselations (Wiley, New York).

Panetta, J.C. (1998) "A mathematical model of drug resistance: Heterogeneous tumors", Mathematical Biosciences 147, 41-61.

Patel, A.A., Gawlinski, E.T., Lemieux, S.K. and Gatenby, R.A. (2001) "A cellular automaton model of early tumor growth and invasion: the effects of native tissue vascularity and increased anaerobic metabolism", Journal of Theoretical Biology 213, 315-331.

Paulus, W. and Peiffer, J. (1989) "Intratumoral histologic heterogeneity of gliomas. A quantitative study", Cancer 64, 442-447.

Poppenborg, H., Munstermann, G., Knopfer, M.M., Hotfilder, M. and Wolff, J.E.A. (1997) "C6 cross-resistant to cisplatin and radiation", Anticancer Research 17, 2073-2077.

Salcman, S., Scholtz, H., Kaplan, R. and Kulik, S. (1994) "Long-term survival in patients with malignant astrocytoma", Neurosurgery $\mathbf{3 4}$ 213-219.

Shah, M.A. and Schwartz, G.K. (2001) "Cell cycle-mediated drug resistance: an emerging concept in cancer chemotherapy", Clinical Cancer Research 7, 2168-2181.

Torquato, S. (1984) "Bulk properties of two-phase media. 1. Cluster expansion for the dielectric constant of dispersions of fully penetrable spheres", Journal of Chemical Physics 81, 5079-5088.

Torquato, S. (2002) Random Heterogeneous Materials: Microstructure and Macroscopic Properties (Springer-Verlag, New York).

Tracqui, P., Cruywagen, G.C., Woodward, D.E., Bartoo, G.T., Murray, J.D. and Alvord, E.C. (1995) "A mathematical model of glioma growth: the effect of chemotherapy on spatio-temporal growth", Cell Proliferation 28, 17-31.

Yung, W.A., Shapiro, J.R. and Shapiro, W.R. (1982) "Heterogeneous chemosensitivities of subpopulations of human glioma cells in culture", Cancer Research 42, 992-998.

Zhang, W., Hara, A., Sakai, N., Andoh, T., Yamada, H., anf, P.H., Gutin, Y.N. and Kornblinth, P.L. (1993) "Radiosensitization and inhibition of deoxyribonucleic acid repair in rat glioma cells by long term treatment with 12-o-tetradecanoylphorbol 13-acatate", Neurosurgery, $432-437$.

\section{APPENDIX}

\section{Determination of $\boldsymbol{R}_{\mathrm{t}}$}

In the proliferation routine of our simulation, the automaton behavior at several steps depends on the overall radius of the tumor, $R_{\mathrm{t}}$. In the present investigation, however, there has been a change in the manner by which 
$R_{\mathrm{t}}$ is determined, as compared to papers I and II. In the previous studies, it was defined as the average radial distance of the proliferative cells at the tumor's outer surface. This method sufficed at the time, as all tumors remained nearly spherical throughout development. In our current work, however, we study certain tumors that deviate from this ideal geometry (see "Results" section, Case 3). Consequently, we choose to define $R_{\mathrm{t}}$ differently. At each step of the simulation, the total tumor volume $\left(V_{\text {tot }}\right)$ is calculated by summing the volumes of every tumor cell (proliferative, non-proliferative and necrotic). The value of $R_{\mathrm{t}}$ is then defined as:

$$
R_{\mathrm{t}}=\left(\frac{3 V_{\mathrm{tot}}}{4 \pi}\right)^{1 / 3} .
$$

Thus, $R_{\mathrm{t}}$ corresponds to the radius that a tumor would adopt if it were distorted to a perfect sphere. With this method of calculation, a tumor's pressure response will depend only upon its volume, not its geometry.

\section{Designation of Localized and Scattered Strains}

For the two-strain tumors of Case 2, scattered and localized resistant strains are designated at the onset of each simulation. The secondary strains are introduced after monoclonal $(\gamma=0.95)$ tumor initialization, but before the onset of the proliferation/treatment algorithm. Computationally, this process occurs in the following manner. For the scattered resistance scenario, every proliferative and non-proliferative cell is checked; for each cell, there exists a probability, $\beta_{0}$, of being converted to the resistant strain. By varying this $\beta_{0}$-term on the microscopic level, we can expect that the same fraction, $\beta_{0}$, of cells will be converted to the resistant strain overall. The process is similar to the mutations of Case 3 .

For the localized resistance scenario, by contrast, each cell is assigned spherical coordinates $(\phi, \theta)$. This $\phi$ is not to be confused with the treatment parameter. The program converts each proliferative and non-proliferative cell whose coordinates exist within a contiguous region to the resistant strain. Specifically, all cells such that $0 \leq \phi \leq X$ and $0 \leq \theta \leq X$ are made resistant; the value of $X$ is varied to yield a particular value of $\beta_{0}$. Finally, following the designation of the secondary strain but prior to the beginning of the proliferation/treatment algorithm, the simulation calculates the explicit value of $\beta_{0}$ to confirm that it is as desired. This confirmation step is executed for both the localized and scattered scenarios. 


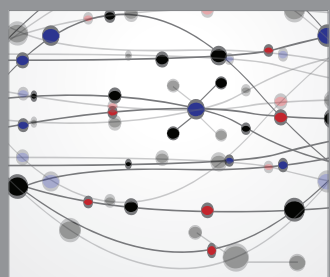

The Scientific World Journal
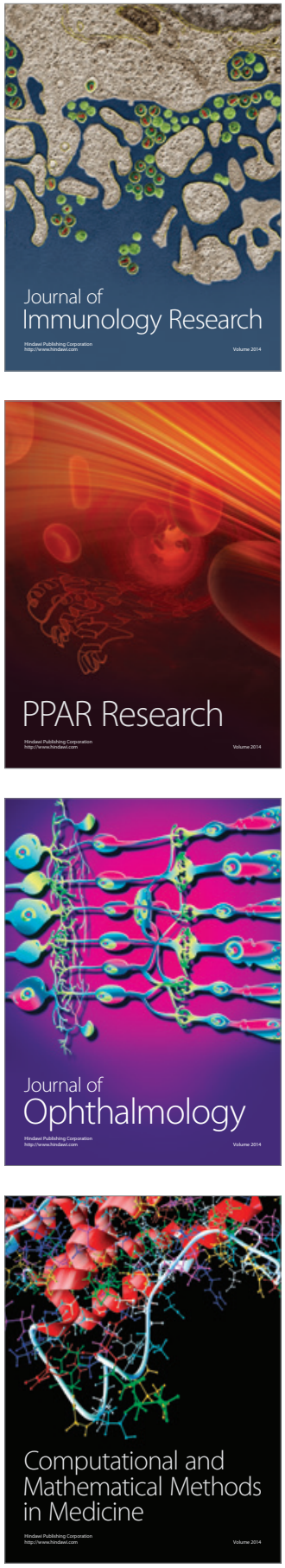

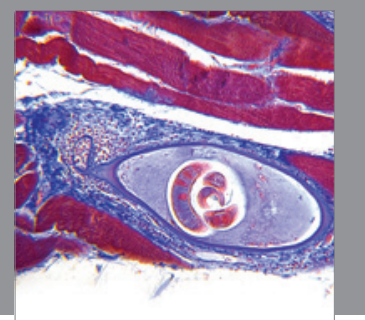

Gastroenterology

Research and Practice
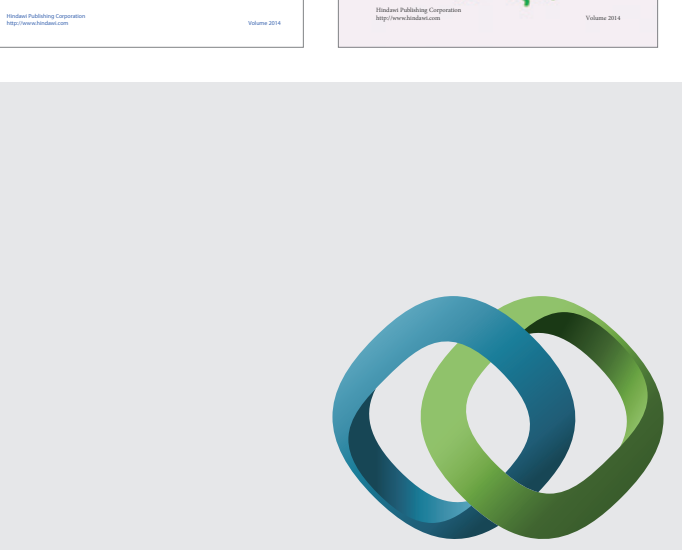

\section{Hindawi}

Submit your manuscripts at

http://www.hindawi.com
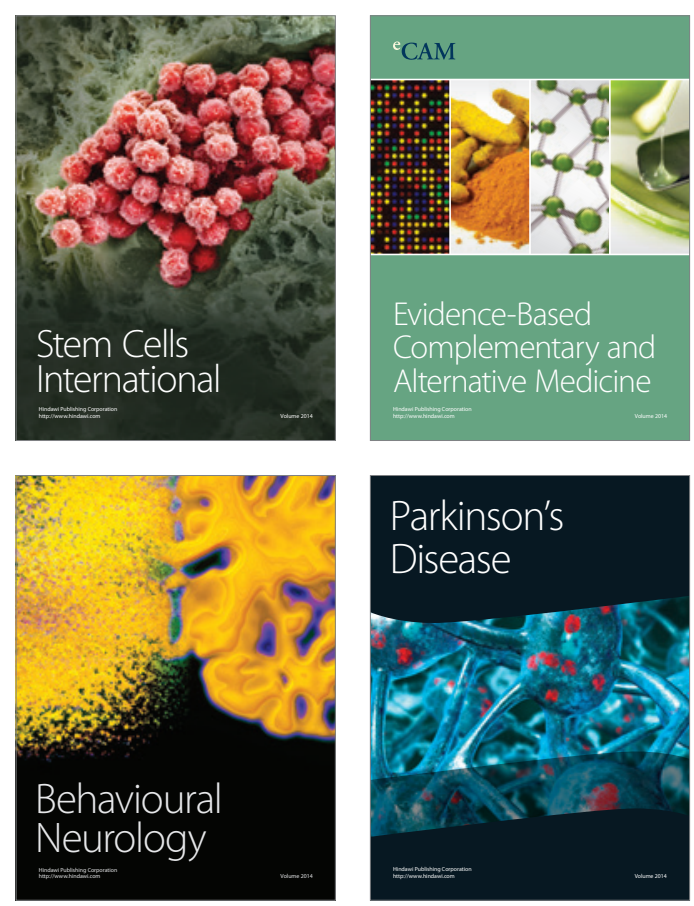

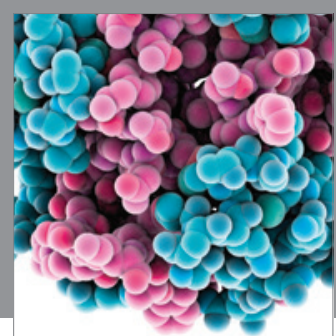

Journal of
Diabetes Research

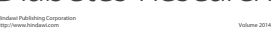

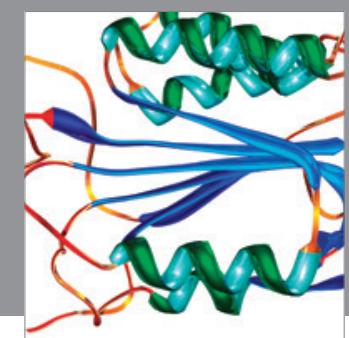

Disease Markers
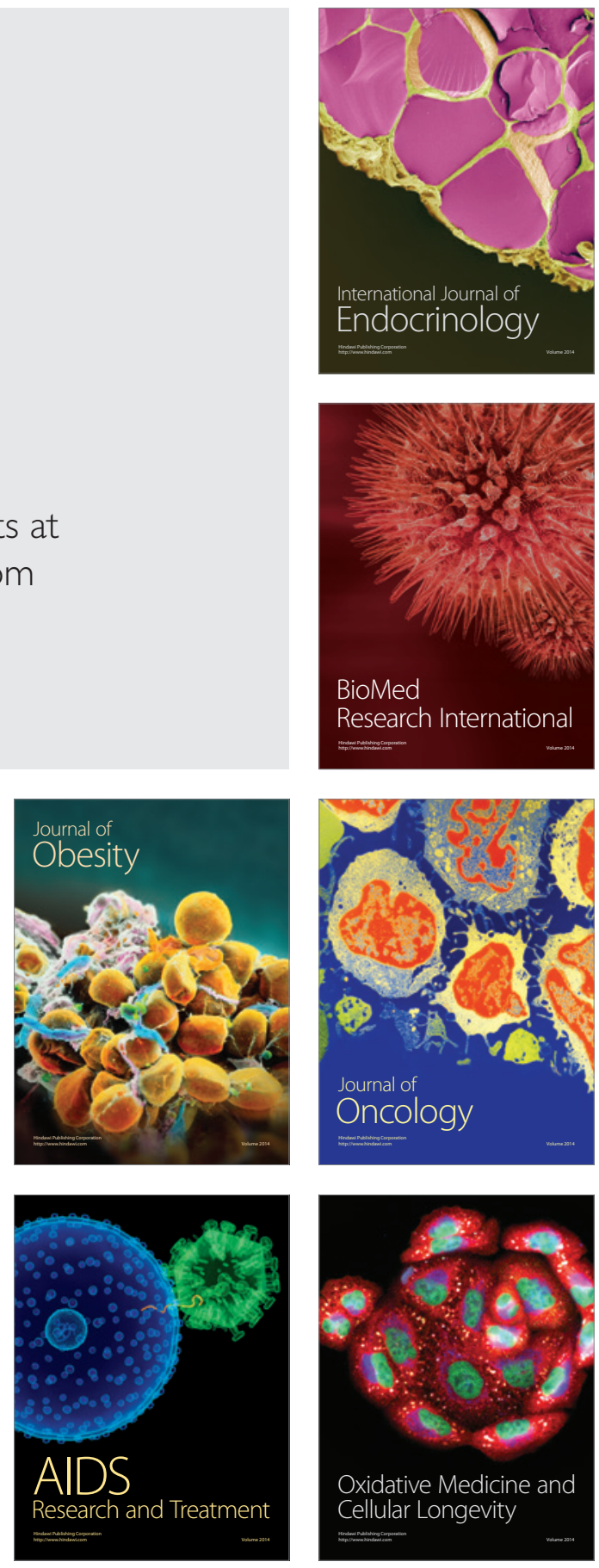